\title{
Non-stationary sound source localization based on zero crossings with the detection of onset intervals
}

\author{
Yong-Jin Park and Hyung-Min Park ${ }^{\text {a) }}$ \\ Department of Electronic Engineering, Sogang University, \\ 1 Shinsu-dong, Mapo-gu, Seoul 121-742, Republic of Korea \\ a) hpark@sogang.ac.kr
}

\begin{abstract}
This paper presents a sound source localization algorithm based on zero crossings in acoustic reverberant environments. From sudden increases of acoustic energy in a non-stationary signal, we identify intervals which dominantly include signal components through direct paths from a source to sensors because they may provide reliable localization cues corresponding to the source direction despite the reverberation. Experimental results show that the presented algorithm which is based on zero crossings with the detection of direct components efficiently accomplishes sound source localization in reverberant environments.
\end{abstract}

Keywords: source localization, zero crossings, binaural processing, interaural time difference

Classification: Science and engineering for electronics

\section{References}

[1] J. W. Strutt, "On our perception of sound direction," Philosophical Magazine, vol. 13, pp. 214-232, 1907.

[2] R. M. Stern and H. Colburn, "Theory of binaural interaction based on auditory-nerve data. IV. A model of subjective lateral position," $J$. Acoust. Soc. Amer., vol. 64, pp. 127-140, 1978.

[3] M. P. Cooke, Modeling Auditory Processing and Organization, Cambridge University Press, Cambridge, U.K., 1993.

[4] Y.-I. Kim and R. M. Kil, "Estimation of interaural time differences based on zero-crossings in noisy multisource environments," IEEE Trans. Audio, Speech, Language Process., vol. 15, pp. 734-743, 2007.

[5] B. Champagne, S. Bedard, and A. Stephenne, "Performance of timedelay estimation in the presence of room reverberation," IEEE Trans. Speech Audio Process., vol. 4, pp. 148-152, 1996.

[6] A. Stephenne and B. Champagne, "A new cepstral prefiltering technique for estimating time delay under reverberant conditions," Signal Process., vol. 59, pp. 253-266, 1997.

[7] J. Benesty, "Adaptive eigenvalue decomposition algorithm for passive acoustic source localization," J. Acoust. Soc. Amer., vol. 107, pp. 384$391,2000$. 
[8] H. M. Park and R. M. Stern, "Spatial separation of speech signals using continuously-variable masks estimated from comparisons of zero crossings," Proc. IEEE ICASSP, Toulouse, France, pp. 1165-1168, May 2006.

[9] B. Shinn-Cunningham, N. Kopco, and T. Martin, "Localizing nearby sound sources in a classroom: binaural room impulse responses," $J$. Acoust. Soc. Amer., vol. 117, pp. 3100-3115, 2005.

[10] J. B. Allen and D. A. Berkley, "Image method for efficiently simulating small-room acoustics," J. Acoust. Soc. Amer., vol. 65, pp. 943-950, 1979.

\section{Introduction}

Sound source localization plays an important role in the human auditory system's ability to select a particular sound source and track the sound originating from that source. It is known that the human auditory system can localize a sound source by exploiting the differences between the signals arriving at the two ears. Especially, interaural time differences (ITDs) and interaural intensity differences (IIDs) serve as the two primary cues for sound source localization. ITDs mainly contribute to localize a source at frequencies below $1.5 \mathrm{kHz}$, and they can also be useful for localization with low-frequency envelopes of higher-frequency components of a sound [1]. IIDs can provide useful information at higher frequencies. Since Jeffress proposed a simple and intuitive mechanism that describes the estimation of ITDs based on interaural coincidences of hypothetical neural activity, many computational models have been developed (e.g. $[2,3])$. Most of these employed a model of peripheral auditory processing which included frequency analysis, a mechanism for estimating the interaural cross-correlation function on a frequencyby-frequency basis, and a mechanism to disambiguate the temporal analysis, typically exploiting IIDs of the signal or consistency over frequency. Recently, Kim et al. estimated ITDs by comparing zero crossings from sensor signals in each frequency band [4]. They demonstrated that it could achieve more robust localization than a cross-correlation-based ITD estimation method in noisy environments with less computational complexity.

Although the zero-crossing-based ITD estimation method can provide the source direction from observations corrupted by diffuse noise, source localization in reverberant environments should be considered for a variety of practical applications. Unfortunately, it is known that acoustic reverberation seriously degrades the localization accuracy, especially due to the presence of virtual sources [5]. Many methods have been suggested to tackle the localization problem, and most of these tried to reduce the effect of reverberation in observations or directly estimate (some aspects of) the reverberant channels (e.g. $[6,7])$. However, they require a number of data and additional computations to accomplish reliable dereverberation or channel estimation.

Note that a typical acoustic impulse response consists of three parts: the direct impulse, early and late reflections. Signal components through direct paths from a source to sensors generate ITDs which correspond to the source 
direction whereas reflection components interfere with the desired ITDs. In this paper, we describe a non-stationary sound source localization method based on zero crossings, which efficiently detects the direct-path components by exploiting characteristics of acoustic impulse responses.

\section{Overall procedure}

Figure 1 illustrates the overall procedure of the proposed algorithm. Input signals are first processed by cochlear filtering for frequency analysis which is simulated by passing each input through a bank of Gammatone filters [3]. The bank consists of 25 channels with center frequencies spaced linearly in the equivalent rectangular bandwidth from $100 \mathrm{~Hz}$ to $4 k \mathrm{~Hz}$. We employed an implementation of the Gammatone filter

$$
g_{i}(t)=t^{3} \exp \left(-2 \pi b_{i} t\right) \cos \left(2 \pi f_{i} t\right) u(t)
$$

as described in [3], where $i, b_{i}, f_{i}$, and $u(t)$ denote the channel index, the decay rate of the impulse response proportional to the bandwidth, the center frequency, and the unit step function, respectively.

The use of zero crossings is motivated by signal processing in the auditory periphery, which represents the synchronous response of low-frequency auditory-nerve fibers to the fine structure of a sound source. We employ a simple ITD estimation method [8]: the zero-crossing time is detected from the filter output of a given sensor, and a search is performed for the closest zero crossing from the other sensor. A zero crossing from a sensor is elaborately estimated by linear interpolation between the two points that straddle the zero crossing. The time difference between zero crossings observed from the two sensors is regarded as a valid estimate of the ITD if it is smaller in magnitude than the time it takes for sound to travel from one sensor to the

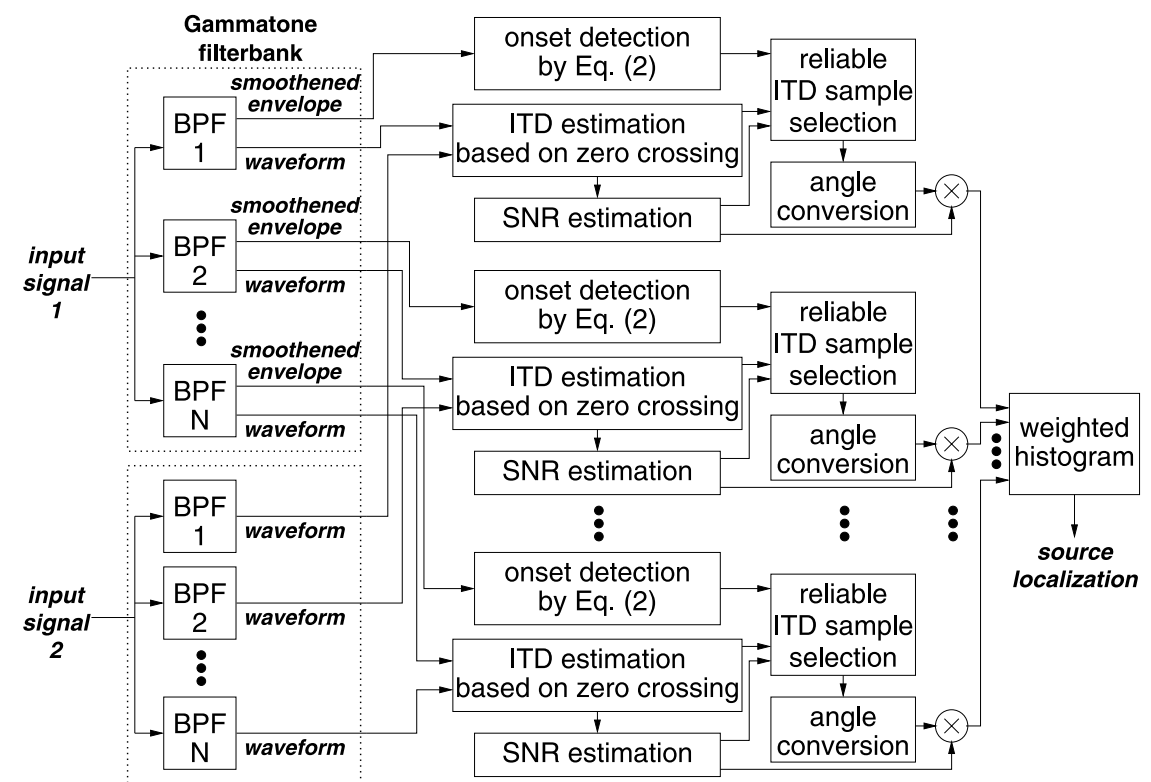

Fig. 1. Overall procedure of the zero-crossing-based source localization for reverberant environments. 
other, and it is discarded otherwise. We make use of closely spaced sensors to avoid spatial aliasing over all frequencies of interest. Hence, the largest possible time difference between the sensors is always less than half the shortest period, so the closest zero crossings provide the desired ITD value.

For sound source localization in reverberant environments, we have to select ITDs from signal components through direct paths from a source to sensors, which correspond to the source direction. Intervals which may dominantly contain the direct components generally correspond to the onset parts of input signal. In order to detect the intervals, the envelope of a Gammatone filter output is employed. The envelope can be obtained by the magnitude of a filter output after replacing the cosine term in Eq. (1) with a complex sinusoid [3]. Since the onsets of a non-stationary signal correspond to sudden increases of acoustic energy, they can be determined by

$$
\frac{E_{i}(t)}{E_{i}(t-\Delta t)}>\mathrm{Th}
$$

where $\Delta t$ represents the time difference between the current and previous envelopes. $E_{i}(t)$ denotes smoothened envelope which is the moving average of envelope values given by

$$
E_{i}(t)=\frac{1}{2 T+1} \sum_{k=-T}^{T} e_{i}(t+k),
$$

where $e_{i}(t)$ is an instantaneous envelope and $T$ determines the number of envelopes to be averaged.

Once an onset is detected by this criterion, we need to elaborate the accurate segment which may provide ITDs corresponding to a source direction. Since direct components of a signal suddenly increase acoustic energy in contrast with reflection components, the beginning of the segment is selected by the time which has the minimum smoothened envelope in an interval with duration $\tau$ before the detected onset time. On the other hand, the end is chosen by the time at the maximum smoothened envelope in an interval with duration $\tau$ after the detected onset time, because a signal after the maximum value may contain many reflection components.

Even though the segment is carefully obtained, it may still contain reflection components. That is because a typical impulse response in an acoustic reverberant environment has so long tail that signal components through late reflections are frequently overlapped with subsequent direct sound. In an acoustic impulse response, late reflections are usually very close to one another and generate different ITDs since a path is different from others. Therefore, the variance of ITDs in a time window may estimate how much the current input signal is contaminated by late reflection components. In order to achieve robustness on diffuse noise, Kim et al. [4] estimated the SNR by employing the variance which is given by

$$
\widehat{\operatorname{SNR}}_{i}(j)= \begin{cases}10 \log _{10} \frac{1}{w_{i}^{2} S_{i}^{2}(j)} & \text { if } w_{i}^{2} S_{i}^{2}(j)<1, \\ 0 & \text { otherwise }\end{cases}
$$


where $w_{i}$ represents the center frequency of the $i$ th channel in a Gammatone filterbank. Also, $j$ and $S_{i}^{2}(j)$ denote an ITD sample index and the sample variance of the ITD and adjacent ones. Here, we employ the SNR estimation method to discard ITDs which give large variances (corresponding to small SNRs) because they may be affected by reflection components. The remaining ITDs are converted into the corresponding azimuth angles.

Finally, to get a weighted histogram, we identify the bin corresponding to the azimuth angle for each ITD and add its estimated SNR value to the selected bin. The accumulation is repeated for all the remaining ITDs across channels. The peaks in the histogram are searched and the corresponding azimuth angles are identified.

It is known that ITDs can be easily affected by background noise while IIDs can be easily biased especially in reverberant environments [9]. Since the SNR estimation can identify reliable ITDs from observations contaminated by diffuse noise, we employ only the ITDs in our work. In addition, we do not need to use IIDs (which are unreliable in reverberant environments) for phase disambiguation in ITD estimation [4] because sensors are sufficiently close to avoid spatial aliasing. The closely spaced sensors are appropriate for compact implementation, and the estimated ITDs might be more reliable because the distortion between sensor inputs is small.

\section{Experimental results}

To evaluate the described method, we simulated sound source localization for various reverberation. For each reverberation time, we considered 320 utterances by 16 speakers from the TI-DIGIT database and source localizations at the azimuths of $0^{\circ}, 30^{\circ}$, or $60^{\circ}$ which are 1 meter distant from the center of two microphones. Considering the bandwidth of the Gammatone filterbank, we simulated signal measurement at sensors which were nominally separated by $43 \mathrm{~mm}$ to avoid spatial aliasing up to $4 \mathrm{kHz}$. To get a sufficient azimuthal resolution with the close proximity of sensors, source signal was upsampled and convolved with the reverberant filters which simulated acoustics from the source to sensors at the $1,024-\mathrm{kHz}$ sampling rate [10]. At a sensor, the convolved signals were combined and downsampled back to $16 \mathrm{kHz}$ to get an observation. Impulse responses from the source to sensors were generated from a virtual rectangular room of 4 meters by 5 meters by 3 meters (in height). A common height of the source and sensors was 1.1 meters. A wall reflection coefficient was chosen to provide a particular reverberation time.

Figure 2 provides the rates of localizations where errors of estimated angles were less than $3^{\circ}$. We have used the parameter values in Table I to detect signal intervals which dominantly contain direct components. We estimated an SNR for each ITD by calculating the sample variance in a window of 5 zero crossings since an onset interval is usually quite short. Also, histograms were composed of ITDs whose estimated SNRs were greater than $30 \mathrm{~dB}$, and their azimuthal resolution was $1^{\circ}$. For comparison, we also display results from ITD estimation based on a conventional cross-correlation func- 


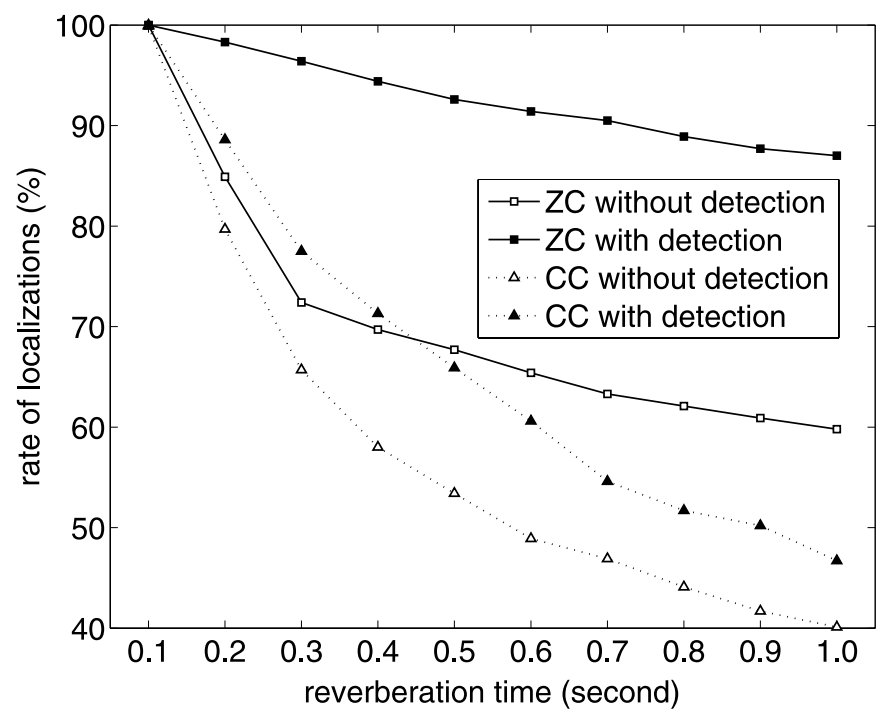

Fig. 2. The rates of localizations where errors of estimated angles were less than $3^{\circ}$.

Table I. The values of parameters to detect onsets.

\begin{tabular}{|c|c|c|c|c|}
\hline Parameter & Th & $\Delta t$ & $T$ & $\tau$ \\
\hline Value & 1.2 & $1 \mathrm{msec}$ & $10 \mathrm{msec}$ & $10 \mathrm{msec}$ \\
\hline
\end{tabular}

tion $[2]^{1}$. Squares display observed rates using localization methods based on zero crossings whereas triangles show those obtained from a conventional cross-correlation function. Filled symbols depict results with the detection of onset intervals described in this paper whereas open symbols represent results without the onset detection. When the cross-correlation-based method employed the onset detection, ITDs were estimated only for frames in which more than half samples belong to the onset intervals. Note that the described algorithm (depicted by a solid line with filled squares) consistently showed better performances than the others. Especially, although an increase in reverberation decreased the rates, the described algorithm was robust to reverberation so that the rate was more than $87.1 \%$ even with the reverberation time of 1 second.

\section{Conclusion}

In this paper, we have described a non-stationary sound source localization method based on zero crossings in reverberant environments. Taking the typical characteristics of acoustic reverberation into account, our approach detects intervals which are dominated by direct-path components, and finds out a desired source direction. Simulation indicated that the described method was capable of robust source localization in reverberant environments.

\footnotetext{
${ }^{1}$ To get a sufficient azimuthal resolution for the cross-correlation-based method, we simulated signal measurements at sensors which were nominally separated by $32 \mathrm{~cm}$. In addition, their sampling rate was $48 \mathrm{kHz}$.
} 


\section{Acknowledgments}

This work was supported by the Sogang University Foundation Research Grants. 